\title{
$N$-酰基- $N$-间氟苄基-6-氨基香豆素的合成及除草活性
}

\author{
王 栋 魏 艳 郝双红* \\ (青岛农业大学化学与药学院 山东省农业仿生应用工程技术研究中心 青岛 266109)
}

\begin{abstract}
摘要 以 $N$-间氟芳基-6-氨基香豆素为先导, 利用活性亚结构拼接原理, 设计、合成了一系列 $N$-酰基- $N$-间氟苄基-6-氨 基香豆素衍生物, 其结构用 ${ }^{1} \mathrm{H}$ NMR、ESI-MS 和元素分析表征, $N$-三氟乙酰基取代的化合物 d5 结构经 X-ray 单晶衍射 进一步确证. 采用琼脂小杯法和盆栽法对合成化合物的除草活性进行了初步研究. 结果表明, $100 \mathrm{mg} / \mathrm{L}$ 分别为 $N$-氯乙 酰基、 $N$-溴乙酰基、 $N$-三氟乙酰基、 $N-(2,4-$ 二氯苯氧乙酰基)取代的化合物 $\mathbf{d} 2, \mathbf{d} \mathbf{3}, \mathbf{d} \mathbf{5}$ 和 $\mathbf{d} 17$ 对反枝苋根、茎的抑制率 大于 $75 \%$, 优于乙草胺 $\mathrm{EC} ; 1500 \mathrm{~g} / \mathrm{ha}$ 分别为 $N$-溴乙酰基、 $N$-(2,4-二氯苯氧乙酰基)取代的化合物 $\mathbf{d} 3$ 和 $\mathbf{d} 17$ 对反枝苋等 双子叶杂草茎叶处理的抑制率达 76\%以上; 同剂量化合物 $\mathbf{d} 17$ 对单子叶作物小麦、玉米和水稻安全.
\end{abstract}

关键词 $N$-酰基- $N$-间氟苄基-6-氨基香豆素衍生物; 合成; 除草活性; 作物安全性

\section{Synthesis and Herbicidal Activity of N-Acyl-N- (m-fluoro-benzyl)-6-amino-coumarin}

\author{
Wang, Dong Wei, Yan Hao, Shuanghong* \\ (College of Chemistry \& Pharmacy, Research Center of Agro-bionic Engineering \& Technology of Shandong Province, \\ Qingdao Agricultural University, Qingdao 266109)
}

\begin{abstract}
Based on the structure of lead compound $N$-( $m$-fluoro-benzyl)-6-amino-coumarin, a series of novel $N$-acyl- $N$-( $m$-fluoro-benzyl)-6-amino-coumarins were designed and synthesized through the principle of bioactive substructure combination. Their structures were characterized by ${ }^{1} \mathrm{H}$ NMR, ESI-MS and elemental analysis. $N$-trifluoroacetyl substituted compound d5 was further confirmed by X-ray single crystal diffraction. The herbicidal activities of synthesized compounds were evaluated as well. The results indicated that compounds substituted respectively by $N$-chloracetyl, $N$-bromoacetyl, $N$-trifluoroacetyl and $N$-(2,4-dichlorophenoxyacetyl) compounds d2, d3, d5 and $\mathbf{d 1 7}$ exhibited marked inhibition against the roots and stems of Amaranthus retroflexus with inhibitory rates above $75 \%$ at $100 \mathrm{mg} / \mathrm{L}$, more active than acetochlor EC. In post-emergence treatment experiment, compounds $\mathbf{d 3}$ and $\mathbf{d 1 7}$ substituted respectively by $N$-bromoacetyl and $N$-(2,4-dichlorophenoxyacetyl) showed more than $76 \%$ inhibition against $A$. retroflexus and other dicotyledons at $1500 \mathrm{~g} /$ ha in greenhouse. Compound $\mathbf{d 1 7}$ is safe to monocotyledonous crops wheat, corn and rice at $1500 \mathrm{~g}$ a.i. $/ \mathrm{hm}^{2}$.

Keywords $N$-acyl- $N$-( $m$-fluoro-benzyl)-6-amino coumarin derivatives; synthesis; herbicidal activity; crop safety
\end{abstract}

天然产物易降解, 作用机理独特, 是研发新型、安 全、高效除草剂先导化合物的重要资源 ${ }^{[1]}$. 香豆素广泛 存在于高等植物, 具有多种优良生物活性 ${ }^{[2 \sim 7]}$, 其除草 活性引人注目 ${ }^{[8,9]}$, 如从 Ferula pseudalliacea 中分离的香 豆素类化合物 sanandajin 和 farnesiferol B 对烟草细胞具 有强烈抑制作用, sanandajin 也可显著抑制杂草种子的 萌发 ${ }^{[10]}$. 实验室合成的4-苯氧基香豆素衍生物可选择性 地控制莎草科等单子叶杂草 ${ }^{[11]}$, 而具三酮结构的 3 -苯甲
酰基-4-羟基香豆素衍生物对油菜有抑制作用 ${ }^{[12]}$.

酰胺类除草剂选择性好、药效高、作用机制多样，在 酰胺类除草剂结构中引入杂环和氟原子是当前此类除 草剂研究开发的热点 ${ }^{[13 \sim 16]}$. 课题组前期研究发现化合 物 $N$-间氟苄基-6-氨基香豆素(c1)对反枝苋种子根、茎生 长具有较强的抑制作用 ${ }^{[17]}$. 为进一步提高活性，本文以 c1 为先导, 根据活性亚结构拼接原理, 引入传统酰胺类 除草剂中的酰化基团，合成了一系列新型 $N$-酰基- $N$-间

\footnotetext{
* E-mail: doublered74@sina.com
}

Received April 7, 2015; revised April 22, 2015; published online April 27, 2015

Project supported by the National Natural Science Foundation of China (No. 31471808) and the Science and Technology Basic Research Project of Qingdao City (No. 12-1-4-5-(9)-jch).

国家自然科学基金(No. 31471808)、青岛市科技计划基础研究(No. 12-1-4-5-(9)-jch)资助项目. 
<smiles>O=c1ccc2ccccc2o1</smiles><smiles>O=c1ccc2cc([N+](=O)[O-])ccc2o1</smiles><smiles>[R]c1cccc(CNc2ccc3oc(=O)ccc3c2)c1</smiles>

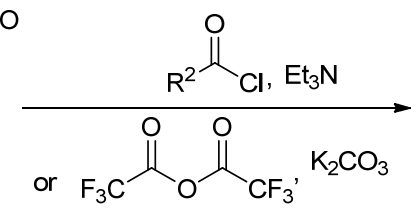

(1)

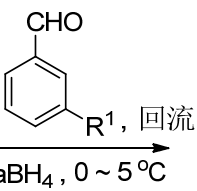

(2) $\mathrm{NaBH}_{4}, 0 \sim 5^{\circ} \mathrm{C}$

b

$\mathrm{R}$<smiles>[R]C(=O)N(Cc1cccc([Z1])c1)c1ccc2oc(=O)ccc2c1</smiles>

d1 d31

\begin{tabular}{|c|c|c|c|c|c|c|c|c|}
\hline Compd. & $\mathrm{R}^{1}$ & $\mathrm{R}^{2}$ & Compd. & $\mathrm{R}^{1}$ & $\mathrm{R}^{2}$ & Comp. & $\mathrm{R}^{1}$ & $\mathrm{R}^{2}$ \\
\hline c1 & $\mathrm{F}$ & & d10 & $\mathrm{F}$ & $\begin{array}{r}\mathrm{CH}_{3} \mathrm{CH}_{2} \mathrm{CH} \\
\mathrm{CH}_{3}\end{array}$ & d21 & $\mathrm{F}$ & $3-\mathrm{ClC}_{6} \mathrm{H}_{4}$ \\
\hline c2 & $\mathrm{CF}_{3}$ & & d11 & $\mathrm{F}$ & $\left(\mathrm{CH}_{3}\right)_{2} \mathrm{CH}$ & d22 & $\mathrm{F}$ & $2,4-\mathrm{ClC}_{6} \mathrm{H}_{3}$ \\
\hline d1 & $\mathrm{F}$ & $\mathrm{CH}_{3}$ & d12 & $\mathrm{F}$ & $\mathrm{CH}_{3} \mathrm{CH}=\mathrm{CH}$ & d23 & $\mathrm{F}$ & 2- $\mathrm{FC}_{6} \mathrm{H}_{4}$ \\
\hline d2 & $F$ & $\mathrm{ClCH}_{2}$ & d13 & $\mathrm{F}$ & $\mathrm{C}_{17} \mathrm{H}_{35}$ & d24 & $\mathrm{F}$ & $3-\mathrm{FC}_{6} \mathrm{H}_{4}$ \\
\hline d3 & $\mathrm{F}$ & $\mathrm{BrCH}_{2}$ & d14 & $\mathrm{F}$ & $\mathrm{CH}_{2}$ & d25 & $\mathrm{F}$ & $3-\mathrm{CF}_{3} \mathrm{C}_{6} \mathrm{H}_{4}$ \\
\hline d4 & $F$ & $\mathrm{CCl}_{3}$ & d15 & $\mathrm{F}$ & $\mathrm{CH}_{2}$ & d26 & $\mathrm{F}$ & $2-\mathrm{NO}_{2} \mathrm{C}_{6} \mathrm{H}_{4}$ \\
\hline d5 & $\mathrm{F}$ & $\mathrm{CF}_{3}$ & d16 & $\mathrm{F}$ & $\mathrm{CH}_{3} \mathrm{OCH}_{2}$ & d27 & $\mathrm{F}$ & 4- $\mathrm{NO}_{2} \mathrm{C}_{6} \mathrm{H}_{4}$ \\
\hline d6 & $\mathrm{F}$ & $\mathrm{CH}_{3} \mathrm{CH}_{2} \mathrm{CH}_{2}$ & d17 & $\mathrm{F}$ & & d28 & $\mathrm{F}$ & \\
\hline d7 & $F$ & $\mathrm{Cl}\left(\mathrm{CH}_{2}\right)_{2} \mathrm{CH}_{2}$ & d18 & $\mathrm{F}$ & & d29 & $\mathrm{F}$ & \\
\hline d8 & $\mathrm{F}$ & $\mathrm{Br}\left(\mathrm{CH}_{2}\right)_{2} \mathrm{CH}_{2}$ & d19 & $\mathrm{F}$ & $\mathrm{Ph}$ & d30 & $\mathrm{F}$ & \\
\hline d9 & $F$ & $\begin{array}{c}\mathrm{CH}_{3} \mathrm{CH}_{2} \mathrm{CH} \\
1 \\
\mathrm{Br}\end{array}$ & d20 & $F$ & $2-\mathrm{ClC}_{6} \mathrm{H}_{4}$ & d31 & $\mathrm{F}$ & \\
\hline
\end{tabular}

图式 1 目标化合物的合成路线

Scheme 1 Synthetic route of target compounds

氟苠基-6-氨基香豆素衍生物，并对其除草活性进行了 初步研究. 其合成路线如图 1 所示.

\section{1 结果与讨论}

\section{1 合成}

采用浓硫酸一硝酸钠合成 $\mathbf{a}^{[18]}$, 容易生成副产物 6,8二硝基香豆素; 浓硫酸-硝酸胍硝化体系合成 $\mathbf{a}$, 产率 高, 产物无需提纯可直接用于下一步反应. 间氟氯茮价 廉, 但与 $\mathbf{b}$ 直接缩合 ${ }^{[19]}$ 易生成二取代副产物, 反应溶剂
及缚酸剂不好选择; 间氟苯甲醛较贵，但以其制备 c1 $^{[17,20]}$, 选择性高, 产率尚好.

\section{2 晶体结构解析}

为进一步确证合成化合物结构, 对 $\mathbf{d 5}$ 进行了 $X$ 射 线单晶衍射分析(CCDC 号为 1060929). 将 d5 溶于乙醇水混合液中，室温静置挥发溶剂，数天后形成淡黄色菱 状晶体. 选取 $0.412 \mathrm{~mm} \times 0.218 \mathrm{~mm} \times 0.063 \mathrm{~mm}$ 单晶 在 $T=296(2) \mathrm{K}, \lambda=0.071073 \mathrm{~nm}$ 下进行结构测试, 在 $\theta$ 为 $3.314^{\circ} \sim 27.569^{\circ}$ 范围内收集衍射点 24627 个, 其中 
$3754[R(\mathrm{int})=0.049]$ 个独立衍射点用于结构的求解和修 正. 结果表明, 化合物 d5 分子式: $\mathrm{C}_{18} \mathrm{H}_{11} \mathrm{~F}_{4} \mathrm{NO}_{3}$, 分子量: 365.28 , 晶型: 单斜晶系, 空间群: $P 21 / n$, 晶胞参数: $a=1.18849(5) \mathrm{nm}, b=1.13960(6) \mathrm{nm}, c=1.25697(6) \mathrm{nm}$, $\alpha=90^{\circ}, \beta=106.5827(14)^{\circ}, \gamma=90^{\circ}, V=1.63164(14) \mathrm{nm}^{3}$, $D_{\mathrm{c}}=1.487 \mathrm{Mg} / \mathrm{m}^{3}, Z=4, F(000)=744, \mu=0.132 \mathrm{~mm}^{-1}$. 晶体结构采用全矩阵最小二乘法修正, 修正后的一致性 因子 $R_{1}=0.1196, w R_{2}=0.3125[I>2 \sigma(I)], R_{1}=0.1594$, $w R_{2}=0.3471$ (所有数据), 修正后残余电子密度: $\Delta \rho_{\max }=$ $1297 \mathrm{e} / \mathrm{nm}^{3}$ 和 $\Delta \rho_{\min }=-956 \mathrm{e} / \mathrm{nm}^{3}$. 化合物 $\mathbf{d 5}$ 分子结构 图见图 2.

\section{3 除草活性测试结果}

\subsection{1 合成化合物除草活性初篣结果}

琼脂小杯法初篮结果(表 1)显示, 部分合成化合物 对马唐、反枝苋种子根、茎的生长具有较强的抑制作用. 2,4-二氯苯氧乙酰基取代的 $\mathbf{d} 17$ 在 $20 \mathrm{mg} / \mathrm{L}$ 剂量下，对反 枝苋种子根、茎的抑制率分别为 $72.3 \%, 75.6 \%, 100 \mathrm{mg} / \mathrm{L}$ 下抑制率为 $97.1 \%, 99.9 \%$, 优于对照药剂乙草胺 EC. 三 氟乙酰基取代的 $\mathbf{d 5}$ 仅对双子叶反枝苋有抑制作用，100 $\mathrm{mg} / \mathrm{L}$ 时，对反枝苋根、茎的抑制率分别为 $78.2 \%, 97.7 \%$, 而对马唐的作用较弱. $100 \mathrm{mg} / \mathrm{L}$ 溴乙酰基取代的 $\mathbf{d 3}$ 对 反枝苋和马唐种子根、茎的抑制率分别为 $80.4 \%, 91.1 \%$ 和 $78.1 \%, 94.1 \%$, 除草活性强于乙草胺 $\mathrm{EC}$ (未列出的化<smiles>O=C(N(Cc1cccc(F)c1)c1ccc2oc(=O)ccc2c1)C(F)(F)F</smiles>

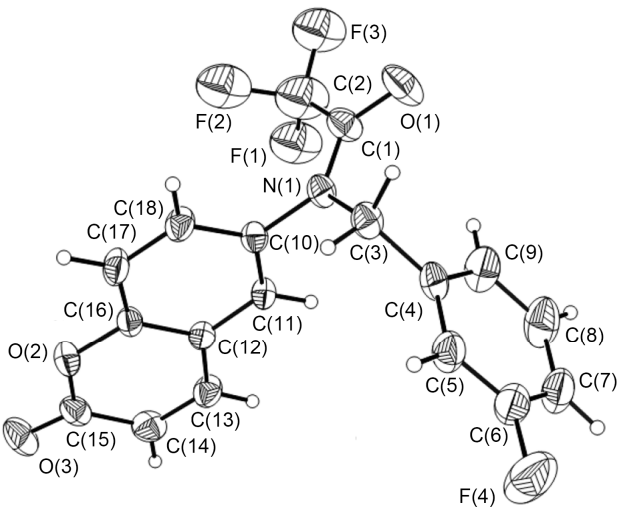

图 2 化合物 $\mathbf{d 5}$ 分子结构图

Figure 2 Molecular structure of compound d5 合物除草活性不明显).

1.3.2 化合物 d3, d5 和 d17 盆栽除草活性及作物安全 性测定结果

温室盆栽除草测试发现(表 2), 在 $1500 \mathrm{~g} / \mathrm{ha}$ 的剂量 下, $\mathbf{d} 3$ 具有较强的茎叶处理防除效果, 对灰藜、反枝苋

表 1 合成目标化合物的除草活性(抑制率/\%)

Table 1 Herbicidal activities (inhibition rate/\%) of synthetic compounds

\begin{tabular}{|c|c|c|c|c|c|c|c|c|}
\hline \multirow{3}{*}{ 化合物 } & \multicolumn{4}{|c|}{ 马唐 } & \multicolumn{4}{|c|}{ 反枝苋 } \\
\hline & \multicolumn{2}{|c|}{$20 \mathrm{mg} / \mathrm{L}$} & \multicolumn{2}{|c|}{$100 \mathrm{mg} / \mathrm{L}$} & \multicolumn{2}{|c|}{$20 \mathrm{mg} / \mathrm{L}$} & \multicolumn{2}{|c|}{$100 \mathrm{mg} / \mathrm{L}$} \\
\hline & 根 & 茎 & 根 & 茎 & 根 & 茎 & 根 & 茎 \\
\hline c1 & 21.9 & 10.7 & 30.0 & 19.9 & 41.9 & 40.2 & 68.7 & 64.6 \\
\hline c2 & 15.3 & 45.7 & 17.8 & 50.1 & 18.0 & 25.3 & 22.7 & 33.8 \\
\hline d1 & 2.4 & 17.0 & 13.3 & 48.5 & 15.2 & 8.1 & 35.4 & 26.0 \\
\hline d2 & 19.3 & 38.9 & 65.0 & 84.7 & 34.0 & 24.4 & 80.1 & 75.5 \\
\hline d3 & 12.5 & 29.7 & 78.1 & 94.1 & 37.8 & 47.2 & 80.4 & 91.1 \\
\hline d4 & 20.6 & 3.9 & 41.8 & 21.9 & 34.6 & 7.7 & 48.6 & 18.8 \\
\hline d5 & 6.4 & 35.1 & 19.4 & 47.4 & 55.2 & 72.6 & 78.2 & 97.7 \\
\hline d6 & 5.2 & 25.8 & 59.13 & 79.9 & 5.4 & 0.3 & 50.5 & 46.5 \\
\hline d7 & 0.7 & 2.4 & 36.6 & 38.9 & 20.8 & 9.1 & 48.1 & 40.4 \\
\hline d8 & 12.8 & 22.6 & 31.3 & 79.1 & 6.4 & 11.9 & 35.2 & 70.2 \\
\hline d9 & 11.4 & 32.3 & 14.0 & 44.3 & 7.9 & 25.3 & 12.3 & 36.2 \\
\hline d10 & 2.1 & 21.3 & 10.8 & 30.0 & 0.5 & 46.3 & 9.0 & 81.5 \\
\hline d12 & 6.7 & 12.2 & 16.4 & 25.2 & 9.7 & 19.7 & 21.3 & 62.7 \\
\hline d14 & 19.3 & 17.3 & 42.4 & 37.5 & 8.8 & 15.4 & 43.7 & 65.6 \\
\hline d15 & 37.3 & 32.4 & 62.4 & 54.6 & 3.0 & 3.5 & 8.4 & 10.6 \\
\hline d17 & 25.3 & 47.2 & 66.1 & 79.3 & 72.3 & 75.6 & 97.1 & 99.9 \\
\hline d20 & 13.0 & 29.6 & 21.1 & 41.3 & 17.3 & 20.4 & 28.7 & 34.8 \\
\hline d23 & 10.5 & 38.5 & 13.4 & 64.7 & 8.6 & 1.5 & 9.3 & 9.7 \\
\hline d30 & 6.7 & 4.2 & 26.8 & 30.5 & 7.0 & 4.2 & 46.1 & 34.1 \\
\hline d31 & 12.3 & 2.6 & 15.3 & 31.0 & 6.0 & 5.1 & 41.3 & 55.1 \\
\hline 乙草胺 EC & 14.4 & 67.2 & 51.6 & 83.8 & 38.0 & 54.4 & 70.2 & 69.9 \\
\hline
\end{tabular}


表 2 化合物 d3, d5 和 $\mathbf{d 1} 7$ 盆栽除草活性(茎叶处理，抑制率 $/ \%$ )

Table 2 Post-emergence herbicidal activities (post-emergence treatment, inhibition rate/\%) of d3, d5 and 17

\begin{tabular}{|c|c|c|c|c|c|c|c|c|}
\hline 化合物 & 浓度/(g・ha $\left.{ }^{-1}\right)$ & 马唐 & 稗草 & 牛筋草 & 早熟禾 & 反枝苋 & 马齿苋 & 灰藜 \\
\hline \multirow{2}{*}{ d3 } & 500 & 1.4 & 4.8 & 8.6 & 0.2 & 39.4 & 5.6 & 48.3 \\
\hline & 1500 & 20.3 & 15.2 & 47.5 & 0.4 & 76.8 & 20.7 & 86.9 \\
\hline \multirow{2}{*}{ d5 } & 500 & 0.4 & 1.6 & 0.3 & 0.1 & 1.3 & 0.5 & 10.2 \\
\hline & 1500 & 0.8 & 9.8 & 0.7 & 0.1 & 1.8 & 0.9 & 33.1 \\
\hline \multirow{2}{*}{ d17 } & 500 & 5.1 & 6.2 & 4.6 & 0.4 & 98.4 & 47.3 & 50.7 \\
\hline & 1500 & 12.6 & 12.7 & 38.6 & 0.8 & 100 & 88.6 & 89.2 \\
\hline \multirow{2}{*}{$2,4-\mathrm{D}$} & 500 & 0.4 & 0.2 & 0.2 & 0.1 & 100 & 98.4 & 100 \\
\hline & 1500 & 0.8 & 0.6 & 0.5 & 0.3 & 100 & 100 & 100 \\
\hline
\end{tabular}

和牛筋草的抑制率分别为 $86.9 \%, 76.8 \%$ 和 $47.5 \%$. d5 活 性较弱, 可能是因在光照条件下不稳定造成的. d17 在 $500 \mathrm{~g} / \mathrm{ha}$ 的剂量下, 对反枝苋的抑制率为 $98.4 \%$, 与 2,4二氯苯氧乙酸(2,4-D)接近, $1500 \mathrm{~g} / \mathrm{ha}$ 的剂量下, 对双子 叶植物灰藜和马齿苋的抑制率在 $88 \%$ 以上, 对单子叶植 物稗草等作用不明显, 表现出较高的选择性. d3, d5 和 d17 土壤处理无除草活性(未列出), 而乙草胺等均为苗 前土壤处理除草剂 ${ }^{[21]}$, 说明该类化合物不同于常见酰 胺类除草剂.

对化合物 d17 的作物安全性进行了测定, 结果如表 3 所示. 在 $500 \mathrm{~g} / \mathrm{ha}$ 和 $1500 \mathrm{~g} / \mathrm{ha}$ 的剂量下, 化合物 $\mathbf{d 1 7}$ 茎叶处理对双子叶作物大豆、油菜和萝卜均产生了一定 的药害, 而对单子叶作物小麦、玉米和水稻表现出较高 的安全性. 供试剂量下, d17 土壤处理对供试作物均无 抑制作用(未列出).

表 3 化合物 d17 作物安全性(茎叶处理，抑制率/\%)

Table 3 Crop safety (post-emergence treatment, inhibition rate/\%) of compounds $\mathbf{d} 17$

\begin{tabular}{lcc}
\hline 供试作物 & $500 \mathrm{~g} / \mathrm{ha}$ & $1500 \mathrm{~g} / \mathrm{ha}$ \\
\hline 小麦 & 0.4 & 0.9 \\
玉米 & 0.7 & 1.2 \\
大豆 & 12.7 & 40.6 \\
水稻 & 0.5 & 1.0 \\
油菜 & 36.4 & 84.2 \\
萝卜 & 42.1 & 93.5 \\
\hline
\end{tabular}

\section{4 构效关系}

氟啶草酮、氟咯草酮和吡氟酰草胺等是八氢番茄红 素脱氢酶(PDS)抑制剂类除草剂, 均具有较好的白化作 用 ${ }^{[22]}$. 这些 PDS 抑制剂结构多样, 但均包含有 3-三氟甲 基苯基的结构. 将 $\mathbf{c 1}$ 中 3-F 换成 3- $\mathrm{CF}_{3}$ 得 $\mathbf{c 2}$, 除草活性 减弱, 且马唐和反枝苋幼苗均未表现出白化现象, 含 3三氟甲基苯基结构的化合物 $\mathbf{d 1 8 ,} \mathbf{d 2 5}$ 也是如此，三者不 同于以上几种 PDS 抑制剂. 酰化衍生物中, 烷酰基类取 代优于烯酰基、苯甲酰基和杂环酰基类，但随碳链增长， 其除草活性减弱; 烷酰基卤代有利于活性提高, 溴代优 于氯代, 碳链末端卤代强于碳链 $\alpha$ 位, 但碳链末端卤原
子数增加时, 氟代优于氯代; 碳链 $\alpha$ 位烷基取代较卤代 有利; 环烷酰基中环越小活性越高; 烷氧乙酰基活性不 及苯氧乙酰基, 且苯氧乙酰基苯环 2,4 -氯代优于 $3-\mathrm{CF}_{3}$.

\section{2 结论}

本文以 $N$-间氟苄基-6-氨基香豆素为先导，利用活 性亚结构拼接原理，设计合成了一系列新型 $N$-酰基- $N$ 间氟芳基-6-氨基香豆素化合物。除草活性测试表明，部 分化合物对马唐和反枝苋具有优异的除草活性. 100 $\mathrm{mg} / \mathrm{L}$ 时，化合物 $\mathbf{d 2}, \mathbf{d} \mathbf{3}, \mathbf{d 5}$ 和 $\mathbf{d 1 7}$ 对反枝苋根、茎的抑 制率大于 $75 \%$ ，优于乙草胺 EC. 在 $1500 \mathrm{~g} / \mathrm{ha}$ 剂量下, 化合物 d3, d17 对反枝苋等双子叶杂草茎叶处理的抑制 率达 $76 \%$ 以上. 在 $500 \mathrm{~g} / \mathrm{ha}$ 和 $1500 \mathrm{~g} / \mathrm{ha}$ 的剂量下, 化合 物 $\mathrm{d} 17$ 对单子叶作物小麦、玉米和水稻安全. 构效关系 表明, c1 引入取代乙酰基类结构对除草活性的贡献较长 链酰基类、苯甲酰基类和杂环酰基类明显. 化合物进一 步的结构优化和活性评价正在进行中.

\section{3 实验部分}

\section{1 仪器与试剂}

WRS-1B 数字熔点仪(未校正); Bruker Avance DPX 500 超导核磁共振仪 $\left(\mathrm{CDCl}_{3}\right.$ 或 DMSO- $d_{6}$ 为溶剂, TMS 为 内标); Agilent 1100 Series 型高效液相色谱/质谱联机系 统(四极杆); PE2400II-CHNS/O 型元素分析仪; Bruker AXS D8 QUEST X 射线单晶衍射仪. 试剂均为市售分析 纯.

\section{2 实验方法}

\subsubsection{6-硝基香豆素(a)的合成}

$14.6 \mathrm{~g}(0.1 \mathrm{~mol})$ 香豆素和 $150 \mathrm{~mL} \mathrm{H}_{2} \mathrm{SO}_{4}$, 搅拌溶解, $0 \sim 5{ }^{\circ} \mathrm{C}$ 下缓慢加入 $12.2 \mathrm{~g}(0.1 \mathrm{~mol})$ 硝酸胍, 搅拌反应 5 $\mathrm{h}, \mathrm{TLC}$ 跟踪反应. 反应完毕，搅拌下迅速将反应液倒入 $500 \mathrm{~mL}$ 冰水中, 出现白色沉淀，抽滤，干燥，得 6-硝基 香豆素. 白色固体, 收率 98.2\%. m.p. 191 192 ${ }^{\circ} \mathrm{C}$ (文献 值 $\left.{ }^{[17]} 189 \sim 191{ }^{\circ} \mathrm{C}\right) ;{ }^{1} \mathrm{H}$ NMR $\left(500 \mathrm{MHz}, \mathrm{CDCl}_{3}\right) \delta$ : 
$8.40 \sim 8.45$ (m, 2H, PhH), 7.81 (d, $J=9.65 \mathrm{~Hz}, 1 \mathrm{H}, \mathrm{H}-4)$, $7.47(\mathrm{~d}, J=9 \mathrm{~Hz}, 1 \mathrm{H}, \mathrm{PhH}), 6.60(\mathrm{~d}, J=9.60 \mathrm{~Hz}, 1 \mathrm{H}$, $\mathrm{H}-3)$.

\subsubsection{6-氨基香豆素 $(\mathbf{b})$ 的合成}

参考文献[17]制备 6-氨基香豆素. 深黄色固体, 收 率 87.1\%. m.p. $143 \sim 145{ }^{\circ} \mathrm{C}$ (文献值 ${ }^{[17]} 164 \sim 165{ }^{\circ} \mathrm{C}$ ); ${ }^{1} \mathrm{H}$ NMR (500 MHz, TMS/DMSO- $\left.d_{6}\right) \delta: 7.89(\mathrm{~d}, J=9.33 \mathrm{~Hz}$, $1 \mathrm{H}, \mathrm{H}-4), 7.10$ (d, $J=8.85 \mathrm{~Hz}, 1 \mathrm{H}, \mathrm{PhH}), 6.84 \sim 6.86(\mathrm{~m}$, $1 \mathrm{H}, \mathrm{PhH}), 6.74(\mathrm{~s}, 1 \mathrm{H}, \mathrm{PhH}), 6.36(\mathrm{~d}, J=9.64 \mathrm{~Hz}, 1 \mathrm{H}$, $\mathrm{H}-3), 5.27$ (s, 2H, $\mathrm{NH}_{2}$ ).

\subsection{3 $\mathrm{N}$-间氟芳基-6-氨基香豆素 $(\mathbf{c 1})$ 的合成}

参考文献[20], $1.0 \mathrm{~g}$ (6.25 mmol) 6-氨基香豆素溶于 $50 \mathrm{~mL}$ 无水乙醇, 摚拌下缓慢滴加 $25 \mathrm{~mL}$ 含 $0.775 \mathrm{~g}$ (6.25 mmol)间氟苯甲醛的无水乙醇溶液, 回流反应 $4 \mathrm{~h}$. $0 \sim 5{ }^{\circ} \mathrm{C}$ 下, 分批加入 $0.475 \mathrm{~g}(12.5 \mathrm{mmol}) \mathrm{NaBH}_{4}$, 搅拌 反应, TLC 跟踪. 反应完毕, 蒸出乙醇, 乙酸乙酯溶解后 水洗两次, 分液, 有机相用无水硫酸钠干燥, 浓缩得黄 褐色油状液体, 通过柱层析[干法上样; 流动相为 $V($ 乙 酸乙酯) $: V$ (石油醚 $)=1: 3$ ]分离得 $N$-间氟芐基-6-氨基 香豆素. 黄色固体, 收率 80.4\%. m.p. 149 150 ${ }^{\circ} \mathrm{C}$ (文献 值 $\left.{ }^{[17]} 149 \sim 150{ }^{\circ} \mathrm{C}\right) ;{ }^{1} \mathrm{H}$ NMR (500 MHz, $\left.\mathrm{CDCl}_{3}\right) \delta: 7.56$ (d, $J=9.50 \mathrm{~Hz}, 1 \mathrm{H}, \mathrm{H}-4), 7.30 \sim 7.35(\mathrm{~m}, 1 \mathrm{H}, \mathrm{PhH})$, $7.14 \sim 7.18(\mathrm{~m}, 2 \mathrm{H}, \mathrm{PhH}), 7.07 \sim 7.09(\mathrm{~m}, 1 \mathrm{H}, \mathrm{PhH})$, $6.96 \sim 7.00(\mathrm{~m}, 1 \mathrm{H}, \mathrm{PhH}), 6.83 \sim 6.85(\mathrm{~m}, 1 \mathrm{H}, \mathrm{PhH}), 6.55$ (d, $J=2.75 \mathrm{~Hz}, 1 \mathrm{H}, \mathrm{PhH}), 6.37$ (d, $J=9.55 \mathrm{~Hz}, 1 \mathrm{H}, \mathrm{H}-3)$, $4.37\left(\mathrm{~s}, 2 \mathrm{H}, \mathrm{PhCH}_{2}\right), 4.21(\mathrm{~s}, 1 \mathrm{H}, \mathrm{NH})$.

以间三氟甲基苯甲醛为原料, 同法制备 $N$-间三氟 甲基苄基-6-氨基香豆素(c2)：黄色固体，收率 82.8\%. m.p. 95 97 ${ }^{\circ} \mathrm{C} ;{ }^{1} \mathrm{H}$ NMR $\left(500 \mathrm{MHz}, \mathrm{CDCl}_{3}\right) \delta: 7.64$ (d, $J=9.65 \mathrm{~Hz}, 1 \mathrm{H}, \mathrm{H}-4), 7.55 \sim 7.57(\mathrm{~m}, 3 \mathrm{H}, \mathrm{PhH}), 7.46 \sim$ $7.49(\mathrm{~m}, 3 \mathrm{H}, \mathrm{PhH}), 7.16(\mathrm{~d}, J=8.95 \mathrm{~Hz}, 1 \mathrm{H}, \mathrm{PhH}), 6.84 \sim$ $6.86(\mathrm{~m}, 1 \mathrm{H}, \mathrm{PhH}), 6.56$ (d, J=2.88 Hz, 1H, PhH), 6.36 (d, $J=9.43 \mathrm{~Hz}, 1 \mathrm{H}, \mathrm{H}-3), 4.43$ (d, $\left.J=4.95 \mathrm{~Hz}, 2 \mathrm{H}, \mathrm{CH}_{2}\right), 4.30$ (s, $1 \mathrm{H}, \mathrm{NH}$ ); ESI-MS $m / z: 320.1[\mathrm{M}+\mathrm{H}]^{+}$. Anal. calcd for $\mathrm{C}_{17} \mathrm{H}_{12} \mathrm{~F}_{3} \mathrm{NO}_{2}$ : C 63.95, H 3.79, N 4.39; found C 63.82, H 3.86, N 4.27 .

3.2.4 $N$-酰基- $N$ - 间氟芐基-6-氨基香豆素 $(\mathbf{d} 1 \sim \mathbf{d} 4$, d6 d31) 的合成

$0.538 \mathrm{~g}$ (2 mmol) $N$-间氟芐基-6-氨基香豆素, $50 \mathrm{~mL}$ 二氯甲烷和 $2.2 \mathrm{mmol}$ 酰氯, 3 滴三乙胺, 室温搅拌, TLC 跟踪反应. 反应完毕, 加饱和 $\mathrm{Na}_{2} \mathrm{CO}_{3}$ 溶液洗至中性, 水 洗, 分液浓缩, 经柱层析 [干法上样; 流动相为 $V$ (石油 醚)：V(乙酸乙酯 $)=2: 1$ ] 分离得 $N$-酰基 $-N$-间氟苄基-6氨基香豆素 ${ }^{[23]}$.

$N$-乙酰基- $N$-间氟苄基-6-氨基香豆素(d1)：淡黄色
晶体, 收率 93.7\%. m.p. 97 99 ${ }^{\circ} \mathrm{C} ;{ }^{1} \mathrm{H}$ NMR (500 MHz, $\left.\mathrm{CDCl}_{3}\right) \delta: 7.61(\mathrm{~d}, J=9.50 \mathrm{~Hz}, 1 \mathrm{H}, \mathrm{H}-4), 7.32(\mathrm{~d}$, $J=8.55 \mathrm{~Hz}, 1 \mathrm{H}, \mathrm{PhH}), 7.24 \sim 7.25(\mathrm{~m}, 1 \mathrm{H}, \mathrm{PhH}), 7.13 \sim$ $7.18(\mathrm{~m}, 2 \mathrm{H}, \mathrm{PhH}), 6.94 \sim 6.99(\mathrm{~m}, 3 \mathrm{H}, \mathrm{PhH}), 6.47$ (d, $J=$ $9.40 \mathrm{~Hz}, 1 \mathrm{H}, \mathrm{H}-3), 4.89$ (s, 2H, $\left.\mathrm{PhCH}_{2}\right), 1.92$ (s, 3H, $\mathrm{CH}_{3}$ ); ESI-MS $m / z: 312.1[\mathrm{M}+\mathrm{H}]^{+}$. Anal. calcd for $\mathrm{C}_{18} \mathrm{H}_{14} \mathrm{FNO}_{3}$ : C 69.45, H 4.53, N 4.50; found C 69.36, H 4.57, N 4.42.

$N$-氯乙酰基- $N$-间氟苄基-6-氨基香豆素 (d2): 黄色 油状液体, 收率 94.2\%. ${ }^{1} \mathrm{H}$ NMR $\left(500 \mathrm{MHz}, \mathrm{CDCl}_{3}\right) \delta$ : $7.70(\mathrm{~d}, J=9.60 \mathrm{~Hz}, 1 \mathrm{H}, \mathrm{H}-4), 7.24 \sim 7.36(\mathrm{~m}, 4 \mathrm{H}, \mathrm{PhH})$, $6.95 \sim 6.98$ (m, 3H, PhH), 6.48 (d, $J=9.60 \mathrm{~Hz}, 1 \mathrm{H}, \mathrm{H}-3)$, 4.92 (s, $2 \mathrm{H}, \mathrm{PhCH}_{2}$ ), 3.91 (s, $2 \mathrm{H}, \mathrm{CH}_{2}$ ); ESI-MS $m / z: 346.0$ $[\mathrm{M}+\mathrm{H}]^{+}$. Anal. calcd for $\mathrm{C}_{18} \mathrm{H}_{13} \mathrm{ClFNO}_{3}: \mathrm{C} 62.53, \mathrm{H} 3.79$, N 4.05; found C 62.46, H 3.81, N 4.13.

$N$-溴乙酰基- $N$-间氟苠基-6-氨基香豆素 $(\mathbf{d} 3)$ : 黄色 油状液体, 收率 $89.4 \%$. ${ }^{1} \mathrm{H}$ NMR $\left(500 \mathrm{MHz}, \mathrm{CDCl}_{3}\right) \delta$ : 7.68 (d, $J=9.68 \mathrm{~Hz}, 1 \mathrm{H}, \mathrm{H}-4), 7.24 \sim 7.35$ (m, 4H, PhH), $6.95 \sim 6.98(\mathrm{~m}, 3 \mathrm{H}, \mathrm{PhH}), 6.48(\mathrm{~d}, J=9.47 \mathrm{~Hz}, 1 \mathrm{H}, \mathrm{H}-3)$, 4.91 (s, $2 \mathrm{H}, \mathrm{PhCH}_{2}$ ), 3.70 (s, $2 \mathrm{H}, \mathrm{BrCH}_{2}$ ); ESI-MS $m / z$ : $389.9[\mathrm{M}+\mathrm{H}]^{+}$. Anal. calcd for $\mathrm{C}_{18} \mathrm{H}_{13} \mathrm{BrFNO}_{3}$ : C 55.41, H 3.36, N 3.59; found C 55.32, H 3.39, N 3.43.

$N$-三氯乙酰基- $N$-间氟苠基-6-氨基香豆素 (d4): 淡 黄色晶体, 收率 90.2\%. m.p. 135 136 ${ }^{\circ} \mathrm{C} ;{ }^{1} \mathrm{H}$ NMR (500 $\left.\mathrm{MHz}, \mathrm{CDCl}_{3}\right) \delta: 7.57$ (d, $\left.J=9.60 \mathrm{~Hz}, 1 \mathrm{H}, \mathrm{H}-4\right), 7.25 \sim$ $7.33(\mathrm{~m}, 4 \mathrm{H}, \mathrm{PhH}), 6.99 \sim 7.03(\mathrm{~m}, 1 \mathrm{H}, \mathrm{PhH}), 6.95 \sim 6.96$ (m, 2H, PhH), 6.46 (d, J=9.60 Hz, 1H, H-3), 4.99 (s, 2H, $\mathrm{PhCH}_{2}$ ); ESI-MS $m / z: 413.9[\mathrm{M}+\mathrm{H}]^{+}$. Anal. calcd for $\mathrm{C}_{18} \mathrm{H}_{11} \mathrm{Cl}_{3} \mathrm{FNO}_{3}$ : C 52.14, H 2.67, N 3.38; found C 52.09, $\mathrm{H} 2.54$, N 3.46 .

$N$-(正丁酰基)- $N$-间氟苠基-6-氨基香豆素(d6)：淡黄 色晶体, 收率 96.2\%. m.p. 103 105 ${ }^{\circ} \mathrm{C} ;{ }^{1} \mathrm{H}$ NMR(500 $\left.\mathrm{MHz}, \mathrm{CDCl}_{3}\right) \delta: 7.65(\mathrm{~d}, J=9.55 \mathrm{~Hz}, 1 \mathrm{H}, \mathrm{H}-4), 7.32(\mathrm{~d}$, $J=8.60 \mathrm{~Hz}, 1 \mathrm{H}, \mathrm{PhH}), 7.22 \sim 7.26(\mathrm{~m}, 1 \mathrm{H}, \mathrm{PhH}), 7.15 \sim$ 7.17 (m, 2H, PhH), 6.94 6.96 (m, 3H, PhH), 6.47 (d, $J=$ $9.55 \mathrm{~Hz}, 1 \mathrm{H}, \mathrm{H}-3), 4.89$ (s, 2H, $\left.\mathrm{PhCH}_{2}\right), 2.06$ (t, $J=7.45$ $\left.\mathrm{Hz}, 2 \mathrm{H}, \mathrm{CH}_{2}-\mathrm{CO}\right), 1.61 \sim 1.69\left(\mathrm{~m}, 2 \mathrm{H}, \mathrm{CH}_{2}\right), 0.86(\mathrm{t}, J=$ $\left.7.40 \mathrm{~Hz}, 3 \mathrm{H}, \mathrm{CH}_{3}\right)$; ESI-MS m/z: $340.1[\mathrm{M}+\mathrm{H}]^{+}$. Anal. calcd for $\mathrm{C}_{20} \mathrm{H}_{18} \mathrm{FNO}_{3}$ : C 70.78, H 5.35, N 4.13; found C 70.67, H 5.28, N 4.26.

$N$-(4-氯丁酰基)- $N$-间氟苄基-6-氨基香豆素 $(\mathbf{d} 7)$ ：淡 黄色晶体, 收率 91.6\%. m.p. 135 137 ${ }^{\circ} \mathrm{C} ;{ }^{1} \mathrm{H}$ NMR (500 $\left.\mathrm{MHz}, \mathrm{CDCl}_{3}\right) \delta: 7.64(\mathrm{~d}, J=9.60 \mathrm{~Hz}, 1 \mathrm{H}, \mathrm{H}-4), 7.33$ (d, $J=8.50 \mathrm{~Hz}, 1 \mathrm{H}, \mathrm{PhH}), 7.23 \sim 7.27(\mathrm{~m}, 1 \mathrm{H}, \mathrm{PhH}), 7.18 \sim$ $7.20(\mathrm{~m}, 2 \mathrm{H}, \mathrm{PhH}), 6.94 \sim 6.97$ (m, 3H, PhH), 6.47 (d, $J=$ $9.60 \mathrm{~Hz}, 1 \mathrm{H}, \mathrm{H}-3$ ), 4.89 (s, 2H, $\mathrm{PhCH}_{2}$ ), 3.58 (t, $J=6 \mathrm{~Hz}$, 
$\left.2 \mathrm{H}, \mathrm{CH}_{2} \mathrm{Cl}\right), 2.25 \sim 2.28\left(\mathrm{~m}, 2 \mathrm{H}, \mathrm{CH}_{2} \mathrm{CO}\right), 2.08 \sim 2.13(\mathrm{~m}$, $2 \mathrm{H}, \mathrm{CH}_{2}$ ); ESI-MS $m / z: 372.2[\mathrm{M}-\mathrm{H}]^{-}$. Anal. calcd for $\mathrm{C}_{20} \mathrm{H}_{17} \mathrm{ClFNO}_{3}$ : C 64.26, H 4.58, N 3.75; found C 64.34, H 4.46, N 3.68 .

$N$-(4-澳丁酰基)- $N$-间氟芐基-6-氨基香豆素(d8): 黄 色油状液体，收率 $88.4 \%$. ${ }^{1} \mathrm{H}$ NMR $(500 \mathrm{MHz}$, $\left.\mathrm{TMS} / \mathrm{CDCl}_{3}\right) \delta: 7.64(\mathrm{~d}, J=9.47 \mathrm{~Hz}, 1 \mathrm{H}, \mathrm{H}-4), 7.24 \sim 7.34$ (m, 4H, PhH), 6.93 6.97 (m, 3H, PhH), 6.46 (d, $J=9.47$ $\mathrm{Hz}, 1 \mathrm{H}, \mathrm{H}-3), 4.91\left(\mathrm{~s}, 2 \mathrm{H}, \mathrm{PhCH}_{2}\right), 1.25 \sim 1.30(\mathrm{~m}, 2 \mathrm{H}$, $\left.\mathrm{BrCH}_{2}\right), 1.09 \sim 1.12\left(\mathrm{~m}, 2 \mathrm{H}, \mathrm{CH}_{2}-\mathrm{CO}\right), 0.68 \sim 0.70(\mathrm{~m}, 2 \mathrm{H}$, $\mathrm{CH}_{2}$ ); ESI-MS $m / z: 417.9[\mathrm{M}-\mathrm{H}]^{-}$. Anal. calcd for $\mathrm{C}_{20} \mathrm{H}_{17} \mathrm{BrFNO}_{3}$ : C 57.43, H 4.10, N 3.35; found C 57.37, H $4.24, \mathrm{~N} 3.31$.

$N$-(2-澳丁酰基)- $N$-间氟芐基-6-氨基香豆素(d9): 黄 色油状液体, 收率 $86.7 \% .{ }^{1} \mathrm{H}$ NMR $\left(500 \mathrm{MHz}, \mathrm{CDCl}_{3}\right) \delta$ : $7.65(\mathrm{~d}, J=9.50 \mathrm{~Hz}, 1 \mathrm{H}, \mathrm{H}-4), 7.34(\mathrm{~d}, J=8.76 \mathrm{~Hz}, 1 \mathrm{H}$, $\mathrm{PhH}), 7.24 \sim 7.29(\mathrm{~m}, 3 \mathrm{H}, \mathrm{PhH}), 6.94 \sim 7.00(\mathrm{~m}, 3 \mathrm{H}, \mathrm{PhH})$, $6.48(\mathrm{~d}, J=9.65 \mathrm{~Hz}, 1 \mathrm{H}, \mathrm{H}-3), 4.84 \sim 4.96(\mathrm{~m}, 2 \mathrm{H}$, $\left.\mathrm{PhCH}_{2}\right), 3.94(\mathrm{t}, J=7.23 \mathrm{~Hz}, 1 \mathrm{H}, \mathrm{CH}), 2.09 \sim 2.15(\mathrm{~m}, 1 \mathrm{H}$, $\left.\mathrm{CH}_{2}\right), 1.89 \sim 1.95\left(\mathrm{~m}, 1 \mathrm{H}, \mathrm{CH}_{2}\right), 0.92(\mathrm{t}, J=7.27 \mathrm{~Hz}, 3 \mathrm{H}$, $\mathrm{CH}_{3}$ ); ESI-MS $m / z$ : $418.0[\mathrm{M}-\mathrm{H}]^{-}$. Anal. calcd for $\mathrm{C}_{20} \mathrm{H}_{17} \mathrm{BrFNO}_{3}$ : C 57.43, H 4.10, N 3.35; found C 57.49, H $4.23, \mathrm{~N} 3.42$.

$N$-(2-甲基丁酰基)- $N$-间氟芳基-6-氨基香豆素 $(\mathbf{d 1 0})$ : 淡黄色晶体, 收率 95.2\%. m.p. 112 $114{ }^{\circ} \mathrm{C} ;{ }^{1} \mathrm{H}$ NMR $\left(500 \mathrm{MHz}, \mathrm{CDCl}_{3}\right) \delta: 7.62$ (d, $\left.J=9.93 \mathrm{~Hz}, 1 \mathrm{H}, \mathrm{H}-4\right), 7.32$ (d, $J=8.08 \mathrm{~Hz}, 1 \mathrm{H}, \mathrm{PhH}), 7.21 \sim 7.25(\mathrm{~m}, 1 \mathrm{H}, \mathrm{PhH})$, $7.08 \sim 7.14(\mathrm{~m}, 2 \mathrm{H}, \mathrm{PhH}), 6.93 \sim 6.98(\mathrm{~m}, 3 \mathrm{H}, \mathrm{PhH}), 6.47$ (d, $J=9.13 \mathrm{~Hz}, 1 \mathrm{H}, \mathrm{H}-3), 4.82 \sim 4.96\left(\mathrm{~m}, 2 \mathrm{H}, \mathrm{PhCH}_{2}\right)$, $2.23 \sim 2.24(\mathrm{~m}, 1 \mathrm{H}, \mathrm{CH}), 1.72 \sim 1.74\left(\mathrm{~m}, 1 \mathrm{H}, \mathrm{CHCH}_{2}\right)$, $1.34 \sim 1.36\left(\mathrm{~m}, 1 \mathrm{H}, \mathrm{CHCH}_{2}\right), 1.06(\mathrm{~d}, J=6.34 \mathrm{~Hz}, 3 \mathrm{H}$, $\left.\mathrm{CHCH}_{3}\right), 0.82$ (t, $J=6.82 \mathrm{~Hz}, 3 \mathrm{H}, \mathrm{CH}_{3}$ ); ESI-MS $m / z$ : $354.1[\mathrm{M}+\mathrm{H}]^{+}$. Anal. calcd for $\mathrm{C}_{21} \mathrm{H}_{20} \mathrm{FNO}_{3}: \mathrm{C} 71.37, \mathrm{H}$ 5.70, N 3.96; found C 71.49, H 5.62, N 3.85.

$N$ - $(i$-丁酰基)- $N$-间氟苄基-6-氨基香豆素(d11)：淡黄 色晶体, 收率 87.3\%. m.p. 142 144 ${ }^{\circ} \mathrm{C} ;{ }^{1} \mathrm{H}$ NMR $(500$ $\left.\mathrm{MHz}, \mathrm{CDCl}_{3}\right) \delta: 7.59(\mathrm{~d}, J=9.56 \mathrm{~Hz}, 1 \mathrm{H}, \mathrm{H}-4), 7.31(\mathrm{~d}$, $J=8.62 \mathrm{~Hz}, 1 \mathrm{H}, \mathrm{PhH}), 7.21 \sim 7.25(\mathrm{~m}, 1 \mathrm{H}, \mathrm{PhH}), 7.14 \sim$ $7.16(\mathrm{~m}, 1 \mathrm{H}, \mathrm{PhH}), 7.09$ (s, 1H, PhH), 6.91 6.98 (m, 3H, $\mathrm{PhH}), 6.46(\mathrm{~d}, J=9.62 \mathrm{~Hz}, 1 \mathrm{H}, \mathrm{H}-3), 4.86\left(\mathrm{~s}, 2 \mathrm{H}, \mathrm{PhCH}_{2}\right)$, $2.41 \sim 2.46(\mathrm{~m}, 1 \mathrm{H}, \mathrm{CH}), 1.07$ (d, $\left.J=6.68 \mathrm{~Hz}, 6 \mathrm{H}, \mathrm{CH}_{3}\right)$; ESI-MS $m / z$ : $340.1[\mathrm{M}+\mathrm{H}]^{+}$. Anal. calcd for $\mathrm{C}_{20} \mathrm{H}_{18} \mathrm{FNO}_{3}$ : C 70.78, H 5.35, N 4.13; found C 70.62, H 5.31, N 4.26.

$N$-巴豆酰基- $N$-间氟芐基-6-氨基香豆素(d12): 黄色 油状液体, 收率 97.6\%. ${ }^{1} \mathrm{H}$ NMR $\left(500 \mathrm{MHz}, \mathrm{CDCl}_{3}\right) \delta$ : $7.64(\mathrm{~d}, J=9.70 \mathrm{~Hz}, 1 \mathrm{H}, \mathrm{H}-4), 7.32(\mathrm{~d}, J=8.93 \mathrm{~Hz}, 1 \mathrm{H}$, $\mathrm{PhH}), 7.16 \sim 7.26(\mathrm{~m}, 3 \mathrm{H}, \mathrm{PhH}), 7.02 \sim 7.07(\mathrm{~m}, 1 \mathrm{H}, \mathrm{PhH})$, $6.96 \sim 6.98(\mathrm{~m}, 2 \mathrm{H}, \mathrm{PhH}), 6.47(\mathrm{~d}, J=9.31 \mathrm{~Hz}, 1 \mathrm{H}, \mathrm{H}-3)$, $5.68(\mathrm{~d}, J=14.75 \mathrm{~Hz}, 1 \mathrm{H}, \mathrm{O}=\mathrm{CCH}=), 4.96(\mathrm{~s}, 2 \mathrm{H}$, $\left.\mathrm{PhCH}_{2}\right), 4.12(\mathrm{dd}, J=7.19,7.22 \mathrm{~Hz}, 1 \mathrm{H}, \mathrm{CH}=), 1.76(\mathrm{~d}$, $J=6.70 \mathrm{~Hz}, 3 \mathrm{H}, \mathrm{CH}_{3}$ ); ESI-MS $m / z: 338.1[\mathrm{M}+\mathrm{H}]^{+}$. Anal. calcd for $\mathrm{C}_{20} \mathrm{H}_{16} \mathrm{FNO}_{3}$ : C 71.21, H 4.78, N 4.15; found $\mathrm{C}$ 71.34, H 4.65, N 4.09.

$N$-硬脂酰基- $N$-间氟芐基-6-氨基香豆素(d13): 白色 晶体, 收率 87.4\%. m.p. 82 83 ${ }^{\circ} \mathrm{C} ;{ }^{1} \mathrm{H}$ NMR $(500 \mathrm{MHz}$, $\left.\mathrm{CDCl}_{3}\right) \delta: 7.59(\mathrm{~d}, J=9.46 \mathrm{~Hz}, 1 \mathrm{H}, \mathrm{H}-4), 7.31(\mathrm{~d}$, $J=8.63 \mathrm{~Hz}, 1 \mathrm{H}, \mathrm{PhH}), 7.21 \sim 7.24(\mathrm{~m}, 1 \mathrm{H}, \mathrm{PhH}), 7.13(\mathrm{~d}$, $J=8.63 \mathrm{~Hz}, 1 \mathrm{H}, \mathrm{PhH}), 7.09$ (s, 1H, PhH), 6.93 6.97 (m, $3 \mathrm{H}, \mathrm{PhH}), 6.46$ (d, $J=9.46 \mathrm{~Hz}, 1 \mathrm{H}, \mathrm{H}-3), 4.87$ (s, $2 \mathrm{H}$, $\mathrm{PhCH}_{2}$ ), 2.05 (t, $J=7.20 \mathrm{~Hz}, 2 \mathrm{H}, \mathrm{CH}_{2} \mathrm{CO}$ ), 1.60 (s, 4H, $\left.\mathrm{CH}_{2}\right), 1.20 \sim 1.29\left(\mathrm{~m}, 26 \mathrm{H}, \mathrm{CH}_{2}\right), 0.88(\mathrm{t}, J=6.79 \mathrm{~Hz}, 3 \mathrm{H}$, $\mathrm{CH}_{3}$ ). ESI-MS $m / z: 536.2[\mathrm{M}+\mathrm{H}]^{+}$. Anal. calcd for $\mathrm{C}_{34} \mathrm{H}_{46} \mathrm{FNO}_{3}$ : C 76.23, $\mathrm{H} 8.65, \mathrm{~N} 2.61$; found $\mathrm{C} 76.34, \mathrm{H}$ $8.52, \mathrm{~N} 2.71$.

$N$-环丙酰基- $N$-间氟芐基-6-氨基香豆素 $(\mathbf{d 1 4})$ ：淡黄 色晶体, 收率 89.6\%. m.p. 106 108 ${ }^{\circ} \mathrm{C} ;{ }^{1} \mathrm{H}$ NMR (500 $\left.\mathrm{MHz}, \mathrm{CDCl}_{3}\right) \delta: 7.66(\mathrm{~d}, J=9.45 \mathrm{~Hz}, 1 \mathrm{H}, \mathrm{H}-4), 7.24 \sim$ $7.34(\mathrm{~m}, 4 \mathrm{H}, \mathrm{PhH}), 6.93 \sim 6.96$ (m, 3H, PhH), 6.46 (d, $J=$ $9.45 \mathrm{~Hz}, 1 \mathrm{H}, \mathrm{H}-3), 4.92$ (s, 2H, $\left.\mathrm{PhCH}_{2}\right), 1.32(\mathrm{~s}, 1 \mathrm{H}, \mathrm{CH})$, $1.08 \sim 1.10\left(\mathrm{~m}, 2 \mathrm{H}, \mathrm{CH}_{2}\right), 0.68 \sim 0.70\left(\mathrm{~m}, 2 \mathrm{H}, \mathrm{CH}_{2}\right)$; ESI-MS $m / z: 338.1[\mathrm{M}+\mathrm{H}]^{+}$. Anal. calcd for $\mathrm{C}_{20} \mathrm{H}_{16} \mathrm{FNO}_{3}$ : C 71.21, H 4.78, N 4.15; found C 71.12, H 4.64, N 4.27.

$N$-环己酰基- $N$-间氟芐基-6-氨基香豆素(d15): 淡黄 色晶体, 收率 89.3\%. m.p. $120 \sim 122{ }^{\circ} \mathrm{C}$; ${ }^{1} \mathrm{H}$ NMR $(500$ $\left.\mathrm{MHz}, \mathrm{CDCl}_{3}\right) \delta: 7.61(\mathrm{~d}, J=9.53 \mathrm{~Hz}, 1 \mathrm{H}, \mathrm{H}-4), 7.31$ (d, $J=8.58 \mathrm{~Hz}, 1 \mathrm{H}, \mathrm{PhH}), 7.21 \sim 7.26(\mathrm{~m}, 1 \mathrm{H}, \mathrm{PhH}), 7.13 \sim$ 7.15 (m, 1H, PhH), 7.09 (s, 1H, PhH), 6.91 6.97 (m, 3H, $\mathrm{PhH}$ ), 6.48 (d, J=9.53 Hz, 1H, H-3), 4.85 (s, 2H, $\mathrm{PhCH}_{2}$ ), $4.12(\mathrm{dd}, J=6.99,7.31 \mathrm{~Hz}, 1 \mathrm{H}, \mathrm{CH}), 1.56 \sim 1.67(\mathrm{~m}, 8 \mathrm{H}$, $\left.\mathrm{CH}_{2}\right), 0.95 \sim 1.00\left(\mathrm{~m}, 2 \mathrm{H}, \mathrm{CH}_{2}\right)$; ESI-MS $m / z: 380.1[\mathrm{M}+$ $\mathrm{H}]^{+}$. Anal. calcd for $\mathrm{C}_{23} \mathrm{H}_{22} \mathrm{FNO}_{3}: \mathrm{C} 72.81$, H 5.84, N 3.69; found C 72.78, H 5.54, N 3.57.

$N$-甲氧基乙酰基- $N$-间氟苄基-6-氨基香豆素(d16): 黄色油状液体, 收率 94.7\%. ${ }^{1} \mathrm{H}$ NMR $\left(500 \mathrm{MHz}, \mathrm{CDCl}_{3}\right)$ $\delta: 7.63(\mathrm{~d}, J=9.07 \mathrm{~Hz}, 1 \mathrm{H}, \mathrm{H}-4), 7.15 \sim 7.34(\mathrm{~m}, 4 \mathrm{H}$, $\mathrm{PhH}), 6.95 \sim 6.97(\mathrm{~m}, 3 \mathrm{H}, \mathrm{PhH}), 6.48(\mathrm{~d}, J=8.77 \mathrm{~Hz}, 1 \mathrm{H}$, $\mathrm{H}-3$ ), 4.89 (s, $2 \mathrm{H}, \mathrm{PhCH}_{2}$ ), 3.80 (s, $2 \mathrm{H}, \mathrm{OCH}_{2}$ ), 3.36 (s, $\left.3 \mathrm{H}, \mathrm{CH}_{3} \mathrm{O}\right)$; ESI-MS $m / z: 342.1[\mathrm{M}+\mathrm{H}]^{+}$. Anal. calcd for $\mathrm{C}_{19} \mathrm{H}_{16} \mathrm{FNO}_{4}$ : C 66.86, H 4.72, N 4.10; found $\mathrm{C} 66.79, \mathrm{H}$ $4.65, \mathrm{~N} 4.29$. 
$N$-(2,4-二氯苯氧乙酰基)- $N$-间氟芐基-6-氨基香豆素 (d17): 淡黄色晶体, 收率 $92.2 \%$. m.p. $182 \sim 183{ }^{\circ} \mathrm{C} ;{ }^{1} \mathrm{H}$ NMR (500 MHz, $\left.\mathrm{CDCl}_{3}\right) \delta: 7.58(\mathrm{~d}, J=9.55 \mathrm{~Hz}, 1 \mathrm{H}, \mathrm{H}-4)$, $7.30 \sim 7.32(\mathrm{~m}, 2 \mathrm{H}, \mathrm{PhH}), 7.22 \sim 7.27(\mathrm{~m}, 1 \mathrm{H}, \mathrm{PhH})$, $7.17 \sim 7.18(\mathrm{~m}, 2 \mathrm{H}, \mathrm{PhH}), 7.12 \sim 7.14(\mathrm{~m}, 1 \mathrm{H}, \mathrm{PhH})$, $6.93 \sim 6.99(\mathrm{~m}, 3 \mathrm{H}, \mathrm{PhH}), 6.75(\mathrm{~d}, J=8.76 \mathrm{~Hz}, 1 \mathrm{H}, \mathrm{PhH})$, 6.46 (d, $J=9.57 \mathrm{~Hz}, 1 \mathrm{H}, \mathrm{H}-3), 4.89$ (s, 2H, $\left.\mathrm{PhCH}_{2}\right), 4.51$ (s, $2 \mathrm{H}, \mathrm{CH}_{2} \mathrm{CO}$ ); ESI-MS m/z: $471.9[\mathrm{M}+\mathrm{H}]^{+}$. Anal. calcd for $\mathrm{C}_{24} \mathrm{H}_{16} \mathrm{Cl}_{2} \mathrm{FNO}_{4}$ : C 61.03, H 3.41, N 2.97; found $\mathrm{C}$ $61.15, \mathrm{H} 3.54, \mathrm{~N} 2.82$.

$N$-(3-三氟甲基苯氧乙酰基)- $N$-间氟苄基-6-氨基香 豆素(d18): 淡黄色晶体, 收率 97.6\%. m.p. $167 \sim 169{ }^{\circ} \mathrm{C}$; ${ }^{1} \mathrm{H}$ NMR $\left(500 \mathrm{MHz}, \mathrm{CDCl}_{3}\right) \delta: 7.59(\mathrm{~d}, J=9.66 \mathrm{~Hz}, 1 \mathrm{H}$, $\mathrm{H}-4), 7.34 \sim 7.38(\mathrm{~m}, 3 \mathrm{H}, \mathrm{PhH}), 7.15 \sim 7.28(\mathrm{~m}, 3 \mathrm{H}, \mathrm{PhH})$, 6.92 7.01 (m, 3H, PhH), 6.48 (d, $J=9.66 \mathrm{~Hz}, 1 \mathrm{H}, \mathrm{H}-3)$, 4.90 (s, $2 \mathrm{H}, \mathrm{PhCH}_{2}$ ), 4.48 (s, $2 \mathrm{H}, \mathrm{CH}_{2} \mathrm{C}=\mathrm{O}$ ); ESI-MS $m / z$ : 472.0 $[\mathrm{M}+\mathrm{H}]^{+}$. Anal. calcd for $\mathrm{C}_{25} \mathrm{H}_{17} \mathrm{~F}_{4} \mathrm{NO}_{4}: \mathrm{C} 63.70, \mathrm{H}$ 3.63, N 2.97; found C 63.52, H 3.48, N 2.89.

$N$-苯甲酰基- $N$-间氟芐基-6-氨基香豆素 $(\mathbf{d 1 9})$ ：淡黄 色晶体, 收率 $98.4 \%$. m.p. $167 \sim 168{ }^{\circ} \mathrm{C} ;{ }^{1} \mathrm{H}$ NMR (500 $\left.\mathrm{MHz}, \mathrm{CDCl}_{3}\right) \delta: 7.46(\mathrm{~d}, J=9.60 \mathrm{~Hz}, 1 \mathrm{H}, \mathrm{H}-4), 7.33$ (d, $J=7.33 \mathrm{~Hz}, 2 \mathrm{H}, \mathrm{PhH}), 7.25 \sim 7.29(\mathrm{~m}, 2 \mathrm{H}, \mathrm{PhH}), 7.18 \sim$ 7.21 (m, 2H, PhH), $7.09 \sim 7.14$ (m, 2H, PhH), 7.05 (d, $J=$ $7.50 \mathrm{~Hz}, 2 \mathrm{H}, \mathrm{PhH}), 6.95 \sim 7.00$ (m, 2H, PhH), 6.37 (d, $J=$ $9.60 \mathrm{~Hz}, 1 \mathrm{H}, \mathrm{H}-3), 5.13\left(\mathrm{~s}, 2 \mathrm{H}, \mathrm{PhCH}_{2}\right)$; ESI-MS $m / z$ : $374.1[\mathrm{M}+\mathrm{H}]^{+}$. Anal. calcd for $\mathrm{C}_{23} \mathrm{H}_{16} \mathrm{FNO}_{3}: \mathrm{C} 73.99, \mathrm{H}$ 4.32, N 3.75; found C 73.85, H 4.43, N 3.67.

$N$-(2-氯苯甲酰基)- $N$-间氟苄基-6-氨基香豆素 $(\mathbf{d 2 0})$ : 淡黄色晶体, 收率 $96.5 \%$. m.p. $135 \sim 137{ }^{\circ} \mathrm{C} ;{ }^{1} \mathrm{H}$ NMR $\left(500 \mathrm{MHz}, \mathrm{CDCl}_{3}\right) \delta: 7.49(\mathrm{~d}, J=9.62 \mathrm{~Hz}, 1 \mathrm{H}, \mathrm{H}-4)$, $7.25 \sim 7.29(\mathrm{~m}, 1 \mathrm{H}, \mathrm{PhH}), 7.04 \sim 7.20(\mathrm{~m}, 9 \mathrm{H}, \mathrm{PhH}), 6.97$ (t, $J=7.99 \mathrm{~Hz}, 1 \mathrm{H}, \mathrm{PhH}), 6.34$ (d, $J=9.45 \mathrm{~Hz}, 1 \mathrm{H}, \mathrm{H}-3)$, $5.13\left(\mathrm{~s}, 2 \mathrm{H}, \mathrm{PhCH}_{2}\right.$ ); ESI-MS $m / z$ : $408.0[\mathrm{M}+\mathrm{H}]^{+}$. Anal. calcd for $\mathrm{C}_{23} \mathrm{H}_{15} \mathrm{ClFNO}_{3}$ : C 67.74, H 3.71, N 3.43; found $\mathrm{C}$ 67.59, H 3.62, N 3.51 .

$N$-(3-氯苯甲酰基)- $N$-间氟芐基-6-氨基香豆素 $(\mathbf{d} 21)$ : 淡黄色晶体, 收率 98.5\%. m.p. 162 164 ${ }^{\circ} \mathrm{C} ;{ }^{1} \mathrm{H}$ NMR $\left(500 \mathrm{MHz}, \mathrm{CDCl}_{3}\right) \delta: 7.49$ (d, $\left.J=9.47 \mathrm{~Hz}, 1 \mathrm{H}, \mathrm{H}-4\right), 7.42$ (s, 1H, PhH), 7.24 7.29 (m, 2H, PhH), 7.16 7.18 (m, $1 \mathrm{H}, \mathrm{PhH}), 7.08 \sim 7.11(\mathrm{~m}, 3 \mathrm{H}, \mathrm{PhH}), 6.96 \sim 7.04(\mathrm{~m}, 4 \mathrm{H}$, $\mathrm{PhH}), 6.40$ (d, $J=9.79 \mathrm{~Hz}, 1 \mathrm{H}, \mathrm{H}-3), 5.10$ (s, $2 \mathrm{H}, \mathrm{PhCH}_{2}$ ); ESI-MS $m / z: 408.0[\mathrm{M}+\mathrm{H}]^{+}$. Anal. calcd for $\mathrm{C}_{23} \mathrm{H}_{15} \mathrm{ClFNO}_{3}: \mathrm{C} 67.74, \mathrm{H} 3.71, \mathrm{~N} 3.43$; found $\mathrm{C} 67.62, \mathrm{H}$ 3.56, N 3.59 .

$N$-(2,4-二氯苯甲酰基)- $N$-间氟苄基-6-氨基香豆素 (d22): 黄色油状液体, 收率 95.3\%. ${ }^{1} \mathrm{H}$ NMR $(500 \mathrm{MHz}$, $\left.\mathrm{CDCl}_{3}\right) \delta: 7.49$ (d, $\left.J=9.63 \mathrm{~Hz}, 1 \mathrm{H}, \mathrm{H}-4\right), 7.26 \sim 7.30$ (m, $1 \mathrm{H}, \mathrm{PhH}), 7.22(\mathrm{~d}, J=1.80 \mathrm{~Hz}, 1 \mathrm{H}, \mathrm{PhH}), 7.07 \sim 7.14(\mathrm{~m}$, $7 \mathrm{H}, \mathrm{PhH}), 6.97 \sim 7.00(\mathrm{~m}, 1 \mathrm{H}, \mathrm{PhH}), 6.38(\mathrm{~d}, J=9.59 \mathrm{~Hz}$, 1H, H-3), 5.10 (s, 2H, $\mathrm{PhCH}_{2}$ ). ESI-MS $m / z$ : 441.9 [M+ $\mathrm{H}]^{+}$. Anal. calcd for $\mathrm{C}_{23} \mathrm{H}_{14} \mathrm{Cl}_{2} \mathrm{FNO}_{3}: \mathrm{C} 62.46, \mathrm{H} 3.19, \mathrm{~N}$ 3.17; found $\mathrm{C} 62.32, \mathrm{H} 3.30, \mathrm{~N} 3.24$.

$N$-(2-氟苯甲酰基)- $N$-间氟芐基-6-氨基香豆素(d23): 淡黄色晶体, 收率 96.4\%. m.p. 139 $140{ }^{\circ} \mathrm{C} ;{ }^{1} \mathrm{H}$ NMR $\left(500 \mathrm{MHz}, \mathrm{CDCl}_{3}\right) \delta: 7.47(\mathrm{~d}, J=8.80 \mathrm{~Hz}, 1 \mathrm{H}, \mathrm{H}-4)$, $7.36 \sim 7.38(\mathrm{~m}, 1 \mathrm{H}, \mathrm{PhH}), 7.27 \sim 7.29(\mathrm{~m}, 1 \mathrm{H}, \mathrm{PhH})$, $7.22 \sim 7.24(\mathrm{~m}, 1 \mathrm{H}, \mathrm{PhH}), 7.06 \sim 7.08(\mathrm{~m}, 6 \mathrm{H}, \mathrm{PhH})$, $6.97 \sim 7.00(\mathrm{~m}, 1 \mathrm{H}, \mathrm{PhH}), 6.79 \sim 6.83(\mathrm{~m}, 1 \mathrm{H}, \mathrm{PhH}), 6.36$ (d, $J=9.40 \mathrm{~Hz}, 1 \mathrm{H}, \mathrm{H}-3$ ), 5.12 (s, 2H, $\mathrm{PhCH}_{2}$ ); ESI-MS $m / z: 392.0[\mathrm{M}+\mathrm{H}]^{+}$. Anal. calcd for $\mathrm{C}_{23} \mathrm{H}_{15} \mathrm{~F}_{2} \mathrm{NO}_{3}: \mathrm{C}$ 70.58, H 3.86, N 3.58; found C 70.64, H 3.71, N 3.61.

$N$-(3-氟苯甲酰基)- $N$-间氟芐基-6-氨基香豆素( $(\mathbf{d 2 4})$ : 淡黄色晶体, 收率 97.1\%. m.p. 162 164 ${ }^{\circ} \mathrm{C} ;{ }^{1} \mathrm{H}$ NMR $\left(500 \mathrm{MHz}, \mathrm{CDCl}_{3}\right) \delta: 7.49(\mathrm{~d}, J=9.66 \mathrm{~Hz}, 1 \mathrm{H}, \mathrm{H}-4)$, $7.25 \sim 7.28(\mathrm{~m}, 1 \mathrm{H}, \mathrm{PhH}), 7.15 \sim 7.17(\mathrm{~m}, 2 \mathrm{H}, \mathrm{PhH})$, $7.09 \sim 7.11(\mathrm{~m}, 2 \mathrm{H}, \mathrm{PhH}), 7.02 \sim 7.06(\mathrm{~m}, 4 \mathrm{H}, \mathrm{PhH})$, $6.95 \sim 6.99(\mathrm{~m}, 2 \mathrm{H}, \mathrm{PhH}), 6.39$ (d, $J=9.45 \mathrm{~Hz}, 1 \mathrm{H}, \mathrm{H}-3)$, $5.11\left(\mathrm{~s}, 2 \mathrm{H}, \mathrm{PhCH}_{2}\right)$; ESI-MS m/z: $392.0[\mathrm{M}+\mathrm{H}]^{+}$. Anal. calcd for $\mathrm{C}_{23} \mathrm{H}_{15} \mathrm{~F}_{2} \mathrm{NO}_{3}$ : C 70.58, $\mathrm{H} 3.86, \mathrm{~N} 3.58$; found $\mathrm{C}$ 70.42, H 3.75, N 3.68 .

$N$-(3-三氟甲基苯甲酰基)- $N$-间氟苄基-6-氨基香豆 素(d25): 淡黄色晶体, 收率 98.6\%. m.p. $160 \sim 162{ }^{\circ} \mathrm{C}$; ${ }^{1} \mathrm{H}$ NMR $\left(500 \mathrm{MHz}, \mathrm{CDCl}_{3}\right) \delta: 7.66(\mathrm{~s}, 1 \mathrm{H}, \mathrm{PhH}), 7.52(\mathrm{~d}$, $J=7.85 \mathrm{~Hz}, 1 \mathrm{H}, \mathrm{H}-4), 7.45 \sim 7.49(\mathrm{~m}, 2 \mathrm{H}, \mathrm{PhH}), 7.26 \sim$ $7.33(\mathrm{~m}, 2 \mathrm{H}, \mathrm{PhH}), 7.16 \sim 7.18(\mathrm{~m}, 1 \mathrm{H}, \mathrm{PhH}), 7.09 \sim 7.11$ (m, 1H, PhH), 6.97 $7.05(\mathrm{~m}, 4 \mathrm{H}, \mathrm{PhH}), 6.39$ (d, $J=9.87$ $\mathrm{Hz}, 1 \mathrm{H}, \mathrm{H}-3), 5.13$ (s, 2H, $\mathrm{PhCH}_{2}$ ); ESI-MS $m / z: 442.0$ $[\mathrm{M}+\mathrm{H}]^{+}$. Anal. calcd for $\mathrm{C}_{24} \mathrm{H}_{15} \mathrm{~F}_{4} \mathrm{NO}_{3}: \mathrm{C}$ 65.31, H 3.43, N 3.17; found C 65.45, H 3.51, N 3.28.

$N$-(3-硝基苯甲酰基)- $N$-间氟苠基-6-氨基香豆素 (d26): 淡黄色晶体, 收率 $97.2 \%$. m.p. $234 \sim 236{ }^{\circ} \mathrm{C} ;{ }^{1} \mathrm{H}$ NMR (500 MHz, $\left.\mathrm{CDCl}_{3}\right) \delta: 8.23(\mathrm{~s}, 1 \mathrm{H}, \mathrm{PhH}), 8.12 \sim 8.14$ (m, 1H, PhH), $7.63 \sim 7.65$ (m, 1H, PhH), 7.49 (d, $J=9.48$ $\mathrm{Hz}, 1 \mathrm{H}, \mathrm{H}-4), 7.39 \sim 7.42(\mathrm{~m}, 1 \mathrm{H}, \mathrm{PhH}), 7.28 \sim 7.31(\mathrm{~m}$, $1 \mathrm{H}, \mathrm{PhH}), 7.17 \sim 7.19(\mathrm{~m}, 1 \mathrm{H}, \mathrm{PhH}), 7.10 \sim 7.12(\mathrm{~m}, 1 \mathrm{H}$, $\mathrm{PhH}), 6.98 \sim 7.05(\mathrm{~m}, 4 \mathrm{H}, \mathrm{PhH}), 6.41(\mathrm{~d}, J=9.65 \mathrm{~Hz}, 1 \mathrm{H}$, $\mathrm{H}-3$ ), 5.13 (s, 2H, $\mathrm{PhCH}_{2}$ ); ESI-MS $m / z: 419.0[\mathrm{M}+\mathrm{H}]^{+}$. Anal. calcd for $\mathrm{C}_{23} \mathrm{H}_{15} \mathrm{FN}_{2} \mathrm{O}_{5}$ : C 66.03, $\mathrm{H}$ 3.61, $\mathrm{N}$ 6.70; found $\mathrm{C} 66.36, \mathrm{H} 3.74, \mathrm{~N} 6.67$.

$\mathrm{N}$-(4-硝基苯甲酰基)- $N$-间氟苄基-6-氨基香豆素 
(d27): 淡黄色晶体, 收率 93.4\%. m.p. 264 265 ${ }^{\circ} \mathrm{C} ;{ }^{1} \mathrm{H}$ NMR $\left(500 \mathrm{MHz}, \mathrm{CDCl}_{3}\right) \delta: 8.06(\mathrm{~d}, J=8.48 \mathrm{~Hz}, 2 \mathrm{H}$, $\mathrm{PhH}), 7.49$ (d, $J=8.17 \mathrm{~Hz}, 2 \mathrm{H}, \mathrm{PhH}), 7.45$ (d, $J=9.95 \mathrm{~Hz}$, $1 \mathrm{H}, \mathrm{H}-4), 7.28 \sim 7.30(\mathrm{~m}, 1 \mathrm{H}, \mathrm{PhH}), 7.17 \sim 7.18(\mathrm{~m}, 1 \mathrm{H}$, $\mathrm{PhH}), 6.98 \sim 7.09(\mathrm{~m}, 5 \mathrm{H}, \mathrm{PhH}), 6.41(\mathrm{~d}, J=9.59 \mathrm{~Hz}, 1 \mathrm{H}$, $\mathrm{H}-3$ ), 5.12 (s, 2H, $\mathrm{PhCH}_{2}$ ); ESI-MS m/z: $419.0[\mathrm{M}+\mathrm{H}]^{+}$. Anal. calcd for $\mathrm{C}_{23} \mathrm{H}_{15} \mathrm{FN}_{2} \mathrm{O}_{5}$ : C 66.03, $\mathrm{H}$ 3.61, N 6.70; found $\mathrm{C} 66.12, \mathrm{H} 3.57, \mathrm{~N} 6.84$.

$N$-(呋喃-2-甲酰基)- $N$-间氟苄基-6-氨基香豆素 (d28): 淡黄色晶体, 收率 86.7\%. m.p. 152 $154{ }^{\circ} \mathrm{C} ;{ }^{1} \mathrm{H}$ NMR (500 MHz, $\left.\mathrm{CDCl}_{3}\right) \delta: 7.61$ (d, $\left.J=9.52 \mathrm{~Hz}, 1 \mathrm{H}, \mathrm{H}-4\right)$, $7.20 \sim 7.30$ (m, 5H, ArH), 7.03 (d, $J=8.02 \mathrm{~Hz}, 2 \mathrm{H}, \mathrm{ArH})$, $6.94 \sim 6.97(\mathrm{~m}, 1 \mathrm{H}, \mathrm{ArH}), 6.45(\mathrm{~d}, J=9.52 \mathrm{~Hz}, 1 \mathrm{H}, \mathrm{H}-3)$, $6.27 \sim 6.28(\mathrm{~m}, 1 \mathrm{H}, \mathrm{ArH}), 6.20(\mathrm{~s}, 1 \mathrm{H}, \mathrm{ArH}), 5.05(\mathrm{~s}, 2 \mathrm{H}$, $\mathrm{PhCH}_{2}$ ); ESI-MS m/z: $364.1[\mathrm{M}+\mathrm{H}]^{+}$. Anal. calcd for $\mathrm{C}_{21} \mathrm{H}_{14} \mathrm{FNO}_{4}$ : C 69.42, H 3.88, N 3.85; found $\mathrm{C} 69.35, \mathrm{H}$ $3.65, \mathrm{~N} 3.92$.

$N$-(噻吩-2-甲酰基)- $N$ - 间氟苠基-6-氨基香豆素 (d29): 淡黄色晶体, 收率 $85.1 \%$. m.p. $141 \sim 143{ }^{\circ} \mathrm{C} ;{ }^{1} \mathrm{H}$ NMR (500 MHz, $\left.\mathrm{CDCl}_{3}\right) \delta: 7.59$ (d, $\left.J=9.64 \mathrm{~Hz}, 1 \mathrm{H}, \mathrm{H}-4\right)$, $7.22 \sim 7.34$ (m, 5H, ArH), 7.04 (d, J=7.90 Hz, 2H, ArH), $6.94 \sim 6.98(\mathrm{~m}, 2 \mathrm{H}, \mathrm{ArH}), 6.83 \sim 6.85(\mathrm{~m}, 1 \mathrm{H}, \mathrm{ArH}), 6.44$ (d, $J=9.36 \mathrm{~Hz}, 1 \mathrm{H}, \mathrm{H}-3), 5.06$ (s, 2H, $\mathrm{PhCH}_{2}$ ); ESI-MS $m / z: 380.0[\mathrm{M}+\mathrm{H}]^{+}$. Anal. calcd for $\mathrm{C}_{21} \mathrm{H}_{14} \mathrm{FNO}_{3} \mathrm{~S}: \mathrm{C}$ 66.48, H 3.72, N 3.69; found C 66.12, H 3.64, N 3.74.

$N$-烟酰基- $N$-间氟苄基-6-氨基香豆素 $(\mathbf{d 3 0})$ : 淡黄色 晶体, 收率 93.7\%. m.p. $155 \sim 157{ }^{\circ} \mathrm{C} ;{ }^{1} \mathrm{H}$ NMR (500 $\left.\mathrm{MHz}, \mathrm{CDCl}_{3}\right) \delta: 8.54(\mathrm{~s}, 1 \mathrm{H}, \mathrm{ArH}), 8.48 \sim 8.50(\mathrm{~m}, 1 \mathrm{H}$, ArH), 7.68 (d, $J=7.64 \mathrm{~Hz}, 1 \mathrm{H}, \operatorname{ArH}), 7.47$ (d, $J=9.82 \mathrm{~Hz}$, $1 \mathrm{H}, \mathrm{H}-4), 7.26 \sim 7.30(\mathrm{~m}, 2 \mathrm{H}, \mathrm{ArH}), 7.16 \sim 7.19(\mathrm{~m}, 2 \mathrm{H}$, $\operatorname{ArH}), 6.97 \sim 7.05(\mathrm{~m}, 4 \mathrm{H}, \mathrm{ArH}), 6.40(\mathrm{~d}, J=9.82 \mathrm{~Hz}, 1 \mathrm{H}$, $\mathrm{H}-3$ ), 5.13 (s, 2H, $\mathrm{PhCH}_{2}$ ); ESI-MS m/z: $375.1[\mathrm{M}+\mathrm{H}]^{+}$. Anal. calcd for $\mathrm{C}_{22} \mathrm{H}_{15} \mathrm{FN}_{2} \mathrm{O}_{3}$ : C 70.58, $\mathrm{H}$ 4.04, $\mathrm{N} 7.48$; found $\mathrm{C} 70.45, \mathrm{H} 4.18, \mathrm{~N} 7.61$.

$N$-(2-氯烟酰基)- $N$-间氟芳基-6-氨基香豆素(d31): 淡黄色晶体, 收率 92.6\%. m.p. 124 $126{ }^{\circ} \mathrm{C} ;{ }^{1} \mathrm{H}$ NMR $\left(500 \mathrm{MHz}, \mathrm{CDCl}_{3}\right) \delta: 8.24 \sim 8.25(\mathrm{~m}, 1 \mathrm{H}, \mathrm{ArH}), 7.52(\mathrm{~d}$, $J=7.90 \mathrm{~Hz}, 1 \mathrm{H}, \mathrm{ArH}), 7.48$ (d, $J=9.60 \mathrm{~Hz}, 1 \mathrm{H}, \mathrm{H}-4)$, $7.28 \sim 7.32(\mathrm{~m}, 1 \mathrm{H}, \mathrm{ArH}), 7.07 \sim 7.14(\mathrm{~m}, 6 \mathrm{H}, \mathrm{ArH})$, $7.00 \sim 7.03$ (m, 1H, ArH), 6.38 (d, $J=9.60 \mathrm{~Hz}, 1 \mathrm{H}, \mathrm{H}-3)$, $5.11\left(\mathrm{~s}, 2 \mathrm{H}, \mathrm{PhCH}_{2}\right)$; ESI-MS m/z: $409.1[\mathrm{M}+\mathrm{H}]^{+}$. Anal. calcd for $\mathrm{C}_{22} \mathrm{H}_{14} \mathrm{ClFN}_{2} \mathrm{O}_{3}$ : C 64.64, H 3.45, N 6.85; found C 64.32, H 3.65, N 6.46.
3.2.5 $N$-三氟乙酰基- $N$-间氟芐基-6-氨基香豆素(d5) 的合成

将 $0.462 \mathrm{~g}(2.2 \mathrm{mmol})$ 三氟乙酸酐, $0.538 \mathrm{~g}(2 \mathrm{mmol})$ $N$-间氟苄基-6-氨基香豆素, $0.069 \mathrm{~g}$ (0.5 mmol) $\mathrm{K}_{2} \mathrm{CO}_{3}$, $40 \mathrm{~mL}$ 二氯甲烷, $0.064 \mathrm{~g}(0.2 \mathrm{mmol}) \mathrm{TBAB}$ 加入到三口 瓶中, 搅拌回流 $6 \mathrm{~h}$, TLC 跟踪反应. 反应完毕, 加饱和 $\mathrm{Na}_{2} \mathrm{CO}_{3}$ 溶液洗至中性, 水洗, 分液, 有机相加无水硫酸 钠干燥, 经重结晶(乙醇-水)得 $N$-三氟乙酰基- $N$-间氟苄

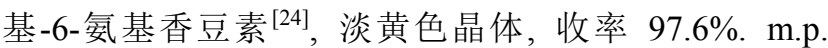
115 $117{ }^{\circ} \mathrm{C} ;{ }^{1} \mathrm{H}$ NMR (500 MHz, $\left.\mathrm{CDCl}_{3}\right) \delta: 7.60(\mathrm{~d}, J=$ $9.60 \mathrm{~Hz}, 1 \mathrm{H}, \mathrm{H}-4), 7.26 \sim 7.33(\mathrm{~m}, 2 \mathrm{H}, \mathrm{PhH}), 7.17 \sim 7.20$ $(\mathrm{m}, 1 \mathrm{H}, \mathrm{PhH}), 7.14(\mathrm{~s}, 1 \mathrm{H}, \mathrm{PhH}), 7.01 \sim 7.05(\mathrm{~m}, 1 \mathrm{H}$, $\mathrm{PhH}), 6.92 \sim 6.95(\mathrm{~m}, 2 \mathrm{H}, \mathrm{PhH}), 6.48(\mathrm{~d}, J=9.60 \mathrm{~Hz}, 1 \mathrm{H}$, $\mathrm{H}-3), 4.91$ (s, 2H, $\mathrm{PhCH}_{2}$ ); ESI-MS m/z: $366.0[\mathrm{M}+\mathrm{H}]^{+}$. Anal. calcd for $\mathrm{C}_{22} \mathrm{H}_{14} \mathrm{ClFN}_{2} \mathrm{O}_{3}$ : C 59.19, H 3.04, N 3.83; found $\mathrm{C} 59.27, \mathrm{H} 3.12, \mathrm{~N} 3.72$.

\section{3 目标化合物的除草活性测定}

\subsection{1 目标化合物的除草活性初篎}

合成化合物除草活性初篮采用琼脂小杯法 ${ }^{[25] ， 以 ~}$ $900 \mathrm{~g} / \mathrm{L}$ 乙草胺 EC 为对照药剂. 选取单子叶杂草马唐和 双子叶杂草反枝苋为供试杂草.

3.3.2 化合物 d3, d5 和 d17 的盆栽除草活性及作物安 全性测定

目标化合物盆栽除草活性及作物安全性测定方法 参照文献[26]. $36 \%$ 噁草酮 EC 和 2,4-D 分别为土壤处理 和茎叶处理对照药剂. 选取单子叶杂草马唐、稗草、牛 筋草和早熟禾, 双子叶杂草反枝苋、马齿苋和灰藜为供 试杂草. 选取单子叶作物小麦、玉米和水稻，双子叶作 物大豆、油菜和萝卜为供试作物种子.

致谢 青岛农业大学制药工程专业 2012 级潘从蕾同学 参与了部分研究工作，在此表示感谢.

辅助材料(Supporting Information) 化合物的 ${ }^{1} \mathrm{H}$ NMR 原始谱图和化合物 d5 晶体结构参数. 这些材料可以免 费从本刊网站(http://sioc-journal.cn/)上下载.

\section{References}

[1] Su, S. Q. Chin. J. Pestic. 2004, 43, 97 (in Chinese). (苏少泉, 农药, 2004, 43, 97.)

[2] Hou, Q. L.; Yang, Z.G.; Ding, W.; Zhang, Y. Q. Nat. Prod. Res. Dev. 2013, 25, 1461 (in Chinese)

(侯秋莉, 杨振国, 丁伟, 张永强, 天然产物研究与开发, 2013, 25,1461 .)

[3] Wang, D. L.; Dong, Z.; Xu, J.; Li, D. Chin. J. Org. Chem. 2013, 33, 1559 (in Chinese).

(王道林, 董哲, 徐姣, 李帝, 有机化学, 2013, 33, 1559.) 
[4] Chen, H.; Zhou, L. K.; Li, S.; Yao, Y. C.; Gu, Y. J.; Li, C. X.; Li, N.; Meng, M.; Li, X. L. Chin. J. Org. Chem. 2013, 33, 164 (in Chinese).

(陈华, 周利凯, 李帅, 姚玉超, 谷云景, 李春晓, 李娜, 孟明, 李小六, 有机化学, 2013, 33, 164.)

[5] Yuan, M. Y.; Liu, W. H.; Zhang, Y. Q.; Yan, H. F.; Zhang, D. N.; Liu, H. M.; Wang, J. P. Chin. J. Org. Chem. 2013, 33, 1108 (in Chinese).

(袁明月, 刘伟华, 张英群, 问红飞, 张冬暖, 刘卉闵, 王建平, 有机化学, 2013, 33, 1108.)

[6] Liu, Z. C.; Liu, X. X.; Wang, Y. H.; Yang, L. Chin. J. Org. Chem. 2011, 31, 136 (in Chinese).

(刘志昌, 刘晓霞, 王应红, 杨录, 有机化学, 2011, 31, 136.)

[7] Yuan, M. Y.; Zhang, D. N.; Yan, H. F.; Liu, W. H.; Li, D. F.; Zhang, Y. Q. Chin. J. Org. Chem. 2011, 31, 1930 (in Chinese). (袁明月, 张冬暖, 闰红飞, 刘伟华, 李德富, 张英群, 有机化学, 2011, 31, 1930.)

[8] Haig, T. J.; Seal, A. N.; Pratley, J. E.; An, M.; Wu, H. J. Chem. Ecol. 2009, 35, 1129.

[9] Zhang, F. H.; Wei, Y.; Wang, D.; Song, W.; Wang, X. G.; Hao, S. H. Chin. J. Pestic. 2011, 6, 455 (in Chinese).

(张方贺, 魏艳, 王栋, 宋卫, 王现刚, 郝双红, 农药, 2011, 6, 455.)

[10] Dara, D.; Peyman, S.; Faezeh, G.; Ahmad, R. G.; Hossein, M.; Naba, A. Ind. Crops. Prod. 2014, 55, 43.

[11] Alvarado, S. I.; Mark, P. A.; Dalke, B. J.; Riley, E. M. $C N$ 951075926, 1996 [Chem. Abstr. 1996, 137, 12085].

[12] Liu, B.; Xie, L. G.; Xu, X. H.; Li, L. H. Chin. J. Org. Chem. 2011, 31, 2067 (in Chinese). (刘斌，谢龙观，徐效华，李永红，有机化学，2011，31，2067.)

[13] Ding, L.; Fu, Y.; Ye, F. Pestic. Sci. Admin. 2011, 9, 2 (in Chinese). (丁丽, 付颖, 叶非, 农药科学与管理, 2011, 9, 22.)

[14] Liu, Q. X.; Huang, M. Z.; Liu, A. P.; Nie, S. Q.; Lei, M. X.; Ren, Y. G.; Pei, H.; He, L. Y.; Hu, L.; Hu, A. X. Chin. J. Org. Chem. 2014, 34,118 (in Chinese).
(刘祈星, 黄明智, 柳爱平, 聂思桥, 雷满香, 任叶果, 裴晖, 何 丽英, 胡礼, 胡艾希, 有机化学, 2014, 34, 118.)

[15] Liu, B.; Dong, H. B.; Han, J. T.; Xu, Z. H.; Jin, S. H.; Wang, M. A. Chin. J. Org. Chem. 2013, 33, 2358 (in Chinese).

(刘斌, 董宏波, 韩金涛, 徐志红, 金淑惠, 王明安, 有机化学, 2013, 33, 2358.)

[16] Dai, H.; Liu, J. B.; Miao, W. K.; Wu, S. S.; Zhang, X.; Wang, T. T.; Fang, J. X. Chin. J. Org. Chem. 2011, 31, 1943 (in Chinese). (戴红, 刘建兵, 苗文科, 吴珊珊, 张欣, 王婷婷, 方建新, 有机 化学, 2011, 31, 1943.)

[17] Zhang, X. L.; Wei, Y.; Wei, N.C.; Hao, S. H. Chin. J. Pestic. Sci. 2013, 15, 34 (in Chinese).

(张学良, 魏艳, 韦能春, 郝双红, 农药学学报, 2013, 15, 34.)

[18] Qi, G.; Tu, S. Z. Biomass Chem. Eng. 2006, 40, 23 (in Chinese). (祁刚, 屠树滋, 生物质化学工程, 2006, 40, 23.)

[19] Basudeb, B.; Susmita P.; Ashis, K. N. Green Chem. 2009, 11, 1115.

[20] Wang, C.; Jiang, R. S.; Feng, F.; Fu, G. L. Chem. Reag. 2008, 30, 935 (in Chinese).

(王诚, 江润生, 冯锋, 付国良, 化学试剂, 2008, 30, 935.)

[21] Tang, C. C.; Li, Y. C.; Chen, B.; Yang, H. Z.; Jin, G. Y. Pesticide Chemistry, Nankai University Press, Tianjin, 1998, pp. 530 531 (in Chinese).

(唐除痴, 李显迏, 陈涁, 杨华铮, 金桂玉, 农药化学, 南开大学 出版社, 天津, 1998, pp. 530 531.)

[22] Yang, H. Z.; Zou, X. M.; Zhu, Y. Q. Modern Pesticide Chemistry, Chemical Industry Press, Beijing, 2013, pp. 631 637 (in Chinese). (杨华铮, 邹小毛, 朱有全, 现代农药化学, 化学工业出版社, 北 京, 2013, pp. 631 637.)

[23] John, E. F.; Michael, A. M. Can. J. Chem. 1992, 70, 1288

[24] Bergeron, R. J.; McManis, J. S. J. Org. Chem. 1988, 53, 3108.

[25] Ma, H. J.; Zhang, J. H.; Xia, X. D.; Xu, M. H.; Ning, J.; Li, J. H. Pest. Manage. Sci. 2014, 70, 946.

[26] Huang, M. Z.; Luo, F. X.; Mo, H. B.; Ren, Y. G.; Wang, X. G.; Ou, X. M.; Lei, M. X.; Liu, A. P.; Huang, L.; Xu, M. C. J. Agric. Food Chem. 2009, 57, 9585. 